\title{
Analysis of $\mathrm{C}-\mathrm{S}-\mathrm{H}$ gel and cement paste by small-angle neutron scattering
}

\author{
Andrew J. Allen ${ }^{a, *}$, Jeffrey J. Thomas ${ }^{b}$ \\ ${ }^{a}$ Materials Science and Engineering Laboratory, NIST, Gaithersburg, MD 20899, USA \\ ${ }^{\mathrm{b}}$ Department of Civil and Environmental Engineering, Northwestern University, Evanston, IL 60208, USA
}

Received 23 November 2005; accepted 5 September 2006

\begin{abstract}
The role of small-angle X-ray and neutron scattering (SAXS and SANS) in the characterization of cement is briefly reviewed. The unique information obtainable from SANS analysis of $\mathrm{C}-\mathrm{S}-\mathrm{H}$ gel in hydrating cement is compared with that obtainable by other neutron methods. Implications for the nature of $\mathrm{C}-\mathrm{S}-\mathrm{H}$ gel, as detected by SANS, are considered in relation to current models. Finally, the application of the SANS method to cement paste is demonstrated by analyzing the effects of calcium chloride acceleration and sucrose retardation on the resulting hydrated microstructure.
\end{abstract}

(C) 2006 Elsevier Ltd. All rights reserved.

Keywords: A: Acceleration; A: Retardation; A: Hydration; B: Calcium-silicate-hydrate (C-S-H); (B): Small-angle neutron scattering

\section{Introduction}

The quantitative and statistically representative characterization and modeling of hydrating cement microstructures using both small-angle neutron and X-ray scattering (SANS and SAXS) has been pursued by groups from around the world, e.g., [1-17]. When a neutron or X-ray beam passes through a material, a component of the beam may be scattered out of the incident beam direction by a small angle due to heterogeneities within the microstructure. The angular profile of the small-angle scattered (SAS) intensity is effectively a Fourier transform of this microstructure. In principle, the angular width, intensity, and the scattering profile can be analyzed to determine the statistically representative size distribution, volume fraction and form of the scattering features [18]. In practice, the data are interpreted using an appropriate microstructure model, and the microstructure is quantified through the parameters obtained. SAS techniques are particularly useful for investigating calcium-silicate-hydrate $\left(\mathrm{C}-\mathrm{S}-\mathrm{H}^{1}\right)$ gel structure, given that the amorphous nature of $\mathrm{C}-\mathrm{S}-\mathrm{H}$ renders diffraction ineffective.

\footnotetext{
* Corresponding author.

E-mail addresses: andrew.allen@nist.gov (A.J. Allen), jthomas@northwestern.edu (J.J. Thomas).

${ }^{1}$ Cement chemistry notation: $\mathrm{C}=\mathrm{CaO}, \mathrm{S}=\mathrm{SiO}_{2}, \mathrm{H}=\mathrm{H}_{2} \mathrm{O}, \mathrm{CH}=\mathrm{Ca}(\mathrm{OH})_{2}$, etc
}

Also, the gel can be affected by sample preparation, requiring great care to be taken for methods such as electron microscopy. By comparison, the sample preparation required for SAS does not disturb the microstructure. Furthermore, the $\mathrm{C}-\mathrm{S}-\mathrm{H}$ gel structure, which exhibits fractal properties, extends over length scales that are appropriate for SAS.

Quantifying the structure of $\mathrm{C}-\mathrm{S}-\mathrm{H}$ is complicated by the presence of water $(\mathrm{H})$ in various states ranging from $\mathrm{OH}^{-}$groups to trapped liquid in the nanoscale pores. A further complication lies in the presence of a fine calcium hydroxide $(\mathrm{CH})$ phase intermixed with $\mathrm{C}-\mathrm{S}-\mathrm{H}$ [19]. This makes it necessary to determine the fine and coarse $\mathrm{CH}$ structure at length scales from nanometers to tens of micrometers so that the $\mathrm{C}-\mathrm{S}-\mathrm{H}$ can be unambiguously characterized. SANS studies can address these issues in two ways. The strong isotope effect in neutron scattering enables much information to be gained from $\mathrm{H}_{2} \mathrm{O} / \mathrm{D}_{2} \mathrm{O}$ contrast variation studies. The recent development of practical ultra-SANS (USANS) instrument configurations provides access up into the $10-\mu \mathrm{m}$ scale regime [20-22].

The quantitative microstructure analysis of $\mathrm{C}-\mathrm{S}-\mathrm{H}$ gel by SAS methods is of particular interest, especially when the results are compared with those of quasielastic neutron scattering (QENS) which can measure the different states of free and bound water (e.g., [23-25]), and inelastic neutron scattering (INS), which can measure the total amount of $\mathrm{CH}$ formation 
[26]. SAS data from cement exhibit characteristic hallmarks of the $\mathrm{C}-\mathrm{S}-\mathrm{H}$ microstructure from which a picture of the $\mathrm{C}-\mathrm{S}-\mathrm{H}$ gel morphology can be discerned.

\section{Small-angle scattering and the difference between SANS and SAXS}

Fig. 1 shows a schematic of a SANS experiment as found at a reactor-based neutron source where a rotating velocity selector provides an incident neutron beam that is monochromatic within a neutron wavelength band of some $10 \%$ to $15 \%$ around a selected wavelength value. For a pulsed neutron source all wavelengths are utilized and different wavelength components are selected during the analysis by time-of-flight methods. In either case, the small-angle scattering intensity data are measured on a $2 \mathrm{D}$ position-sensitive detector. The geometric angle of scatter between the incident and scattered beam, $2 \theta$, is converted into a scattering vector of magnitude, $Q=(4 \pi / \lambda) \sin \theta)$, where $\lambda$ is the neutron or X-ray wavelength and the direction of $Q$ is as defined below. In general, the scattering from fine microstructure features occurs at relatively large scattering angles or $Q$ values; that from coarse features occurs at small $Q$.

Fig. 2 presents typical SANS data for a hydrating Portland cement. The intensity data shown are normalized to the incident beam intensity and corrected for both sample absorption and other background scattering effects (including a significant flat background subtraction at high $Q$ for both SAXS and SANS), and have been circularly-averaged about the incident beam direction. The result, called the scattering cross-section, $\mathrm{d} \Sigma / \mathrm{d} \Omega$, is a material property (for X-ray or neutron scattering) and is the probability per unit sample volume of scattering into unit solid angle about a direction corresponding to the scattering vector, $\boldsymbol{Q}$ $[10,18]$. The direction of $\boldsymbol{Q}$ bisects the incident and scattered beam directions and, for small scattering angles, is approximately in the plane of the sample (Fig. 1) as well as in that of the incident and scattered beams. On a $\log -\log$ plot of $\mathrm{d} \Sigma / \mathrm{d} \Omega$ versus $Q$ the general shape of the scattering curve from a given microstructure is similar for SAXS and SANS. However, SAXS values of $\mathrm{d} \Sigma / \mathrm{d} \Omega$ are significantly larger than for SANS. SAXS arises due to X-ray photon scattering from whole atoms (or ensembles of atoms) and the scattered intensity varies with the square of the electron number density; SANS arises due to neutron scattering from the atomic nuclei and the scattered intensity fluctuates through the periodic table with some strong isotope effects. In particular, the SANS from hydrogen or $\mathrm{H}_{2} \mathrm{O}$

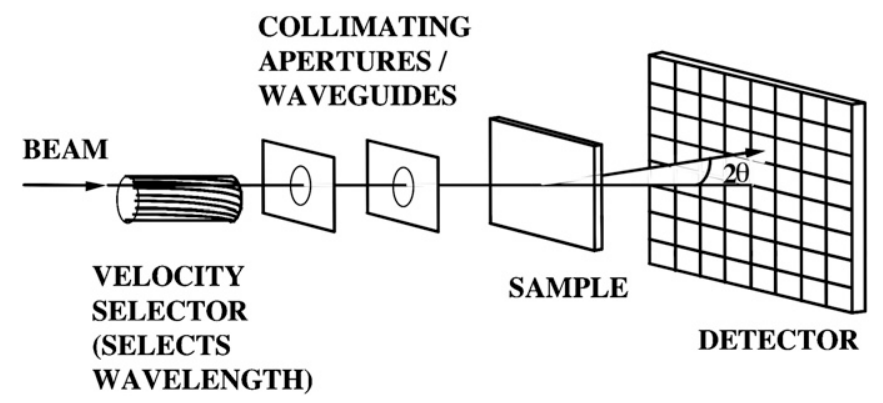

Fig. 1. Schematic of a typical reactor-based SANS instrument.

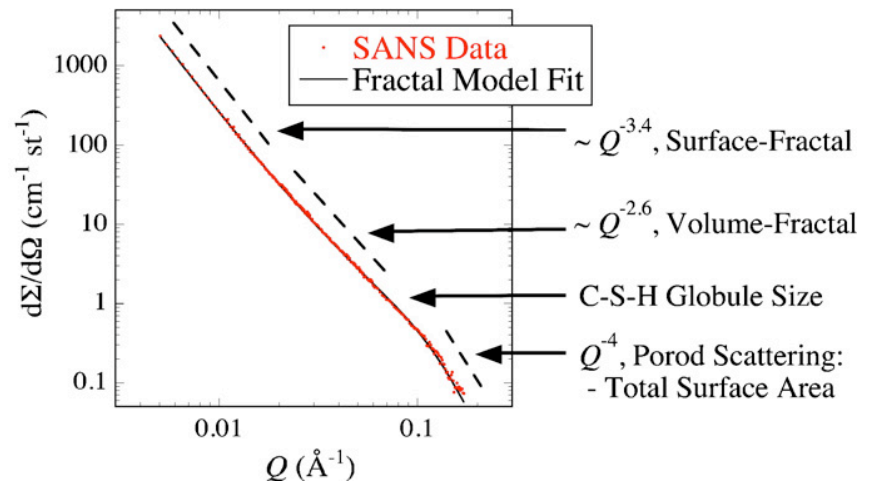

Fig. 2. Typical SANS data for cement paste together with a fractal model fit, as discussed in the text. Major hallmarks are indicated together with their associated microstructure features. The dashed lines have the $Q$ power law exponents indicated for the different $Q$ regimes.

is significantly different to that from deuterium or $\mathrm{D}_{2} \mathrm{O}$ (heavy water) [11]. The neutron scattering contrast for hydrating cement can be varied by changing the hydrogen/deuterium ratio in the pore water, or other pore fluid. Frequently, the scattered intensities are simply referred to as $I(Q)$ but for quantitative comparisons between different experiments, it is essential to establish that the $I(Q)$ data meet the definition requirements for $\mathrm{d} \Sigma / \mathrm{d} \Omega$. Due to the extended $Q$ and intensity ranges involved, $\log -\log$ plots of $I(Q)$ versus $Q$ exhibit rather subtle changes for different cement microstructures, and it is convenient to plot $I(Q) \times Q^{4}$ versus $Q$ on a $\log -\log$ scale for evaluating microstructure differences [15], as shown later.

\section{Analysis of SANS data}

Many microstructure models are now available [27], from use of the Guinier approximation for particulate systems to a full size distribution analysis of coherently ordered microstructures [18], or the characterization of fractal morphologies [4]. Generally, an inverse relationship exists between $Q$ and scattering size. Typically, the data are analyzed to provide a quantitative measure of a model microstructure based on an interpretation established from microscopy. Occasionally, hallmarks appear in the data, associated with particular morphologies. These include peaks when interference effects occur in an ordered or periodic microstructure, $Q^{-1}$ scattering for long capillaries, or $Q^{-2}$ scattering for sheets or membranes [18].

\subsection{Fractal versus other microstructures for $\mathrm{C}-\mathrm{S}-\mathrm{H}$ gel}

It must be stated that the $Q^{-2}$ scattering that would be indicative of a possible sheet-like $\mathrm{C}-\mathrm{S}-\mathrm{H}$ structure, proposed elsewhere (e.g., [19]), has not been observed for cement systems, except where extensive leaching has reduced the overall $\mathrm{Ca} / \mathrm{Si}$ ratio to less than 1.5 [15]. This does not preclude a sheet structure for $\mathrm{C}-\mathrm{S}-\mathrm{H}$ at the nanometer scale if this is folded up within the observed $\mathrm{C}-\mathrm{S}-\mathrm{H}$ microstructure, but it does preclude a sheet-like morphology on a more extensive length scale. Instead, as Fig. 2 indicates, the data can be interpreted using a combination of surface-fractal and mass- or volume-fractal 
components, together with a building block globule size of diameter $5 \mathrm{~nm}$ or less [4,5]. Mass-fractal structures are characterized by non-integral power law $Q^{-\mathrm{D}_{\mathrm{v}}}$ scattering where (usually) $2<D_{\mathrm{V}}<3$; surface-fractal structures are characterized by non-integral power law $Q^{-\left(6-\mathrm{D}_{\mathrm{S}}\right)}$ scattering where $2<D_{\mathrm{S}}<3$ so that the scattering exponent is between 3 and 4 . Our general interpretation of the data in Fig. 2 is that the gel globules and volume-fractal structure comprise the $\mathrm{C}-\mathrm{S}-\mathrm{H}$ low-density or outer-product structure in hydrating cement, and that the surface-fractal structure arises where this decorates clinker grains or other coarse features. [As can be inferred from Fig. 2, both $D_{\mathrm{V}}$ and $D_{\mathrm{S}}$ are frequently close to 2.4 , suggesting that a common structure underlies both fractal morphologies.] A quantitative model can determine not only the fractal exponents, $D_{\mathrm{V}}$ and $D_{\mathrm{S}}$, but also $\mathrm{C}-\mathrm{S}-\mathrm{H}$ volume fractions, surface areas, and the correlation lengths that define the size regimes for fractal scaling [10]. In recent years, this fractal interpretation has proven useful for following cement hydration in real time, for exploring the effects of cement additives (e.g., silica fume), and for assessing the microstructure effects of leaching [4-17]. However, an absolute determination of the microstructure parameters depends not only on absolute calibration of the SANS or SAXS data (i.e. $\mathrm{d} \Sigma / \mathrm{d} \Omega$ ) but also on accurate knowledge of the scattering contrast for $\mathrm{C}-\mathrm{S}-\mathrm{H}$ gel. This issue remains a challenge due to the uncertain nature of $\mathrm{C}-\mathrm{S}-\mathrm{H}$, and is a subject of ongoing research by the authors. SANS contrast variation studies are central to such efforts.

\subsection{Scattering invariant}

In principle, scattering theory provides a method to circumvent uncertainties in the $\mathrm{C}-\mathrm{S}-\mathrm{H}$ scattering contrast [18], through application of the scattering invariant, $Q_{\text {INVARIANT, given by: }}$

$Q_{\text {INVARIANT }}=2 \pi^{2} \Phi_{\mathrm{T}}\left(1-\Phi_{\mathrm{T}}\right)|\Delta \rho|^{2}=\int_{0}^{\infty} Q^{2} \frac{\mathrm{d} \Sigma}{\mathrm{d} \Omega}(Q) \mathrm{d} Q$

where $\Phi_{\mathrm{T}}$ is either the solid volume fraction or the porosity and $|\Delta \rho|^{2}$ is the scattering contrast between the voids and the solid phase. Independent knowledge of the cement porosity, set to $\Phi_{\mathrm{T}}$ or $\left(1-\Phi_{\mathrm{T}}\right)$ affords a value of $|\Delta \rho|^{2}$ if the $Q^{2}$-weighted SANS or SAXS data are integrated across the $Q$-range as indicated in Eq. (1). This approach was taken in early SAXS studies of cement [1]. However, Fig. 3 indicates limitations in this method. The integral must be taken over all $Q$, not just the $Q$-range measured. This implies that an extrapolation must be made both to $Q=0$ and to $Q=\infty$ (or at least, $Q=4 \pi / \lambda$ ). Fig. 3 shows how significant parts of the integral fall outside of the measured $Q$-range. If this is ignored $Q_{\text {INVARIANT }}$ can be underestimated, leading to a spuriously high specific surface area determination if derived from a comparison of Eq. (1) with that for the Porod scattering:

$\frac{\mathrm{d} \Sigma}{\mathrm{d} \Omega}=\frac{2 \pi|\Delta \rho|^{2} S_{\mathrm{T}}}{Q^{4}}$

where $S_{\mathrm{T}}$ is the surface area per unit sample volume. (The unknown $|\Delta \rho|^{2}$ cancels out in the comparison.) Difficulties in

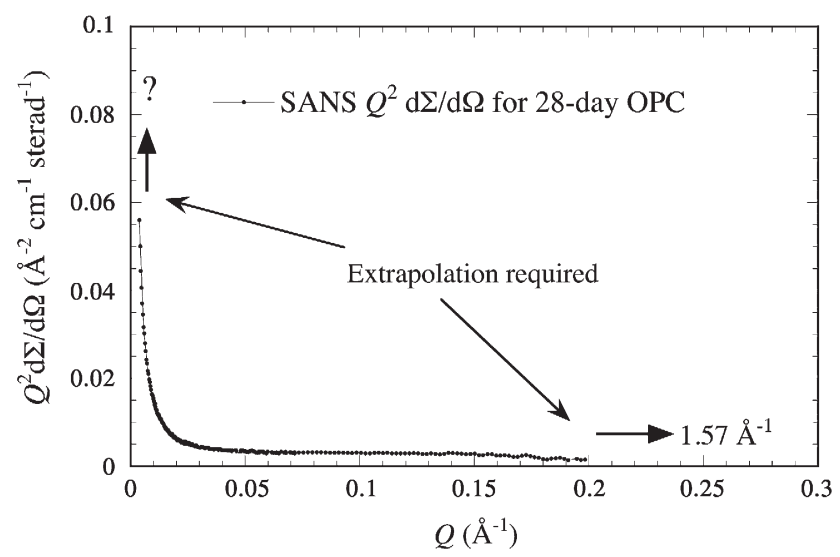

Fig. 3. Measured and extrapolated integrand for scattering invariant with wavelength $0.8 \mathrm{~nm}$ (maximum $Q=15.7 \mathrm{~nm}^{-1}$ or $1.57 \AA^{-1}$ ).

making the data extrapolation arise from uncertainty in the high values of $\mathrm{d} \Sigma / \mathrm{d} \Omega$ at low $Q$, and in the flat background subtraction at high $Q$ (note data are $Q^{2}$-weighted in the integral). Thus, absolute determination of $\mathrm{d} \Sigma / \mathrm{d} \Omega$ and $|\Delta \rho|^{2}$ for both SANS and SAXS is generally preferred over the scattering invariant method for investigations of hydrating cement.

\subsection{USAXS and USANS extend the microstructure size range}

Ultrasmall-angle scattering (USAXS and USANS) instruments apply crystal diffraction optics to obtain SAXS or SANS data to much lower $Q$ values than can be obtained using the conventional geometry shown in Fig. 1 [20-22]. USANS extends the range to particularly low $Q\left(3 \times 10^{-5} \AA^{-1}\right)$. This enables microstructure characterization to be extended to larger feature sizes: to beyond $1 \mu \mathrm{m}$ for USAXS, and to more than $10 \mu \mathrm{m}$ for USANS, — provided the sample can be made sufficiently thin to avoid significant multiple scattering. For cement systems, we have found an ideal coupon thickness of $0.4 \mathrm{~mm}-0.5 \mathrm{~mm}$ for SANS, SAXS, USANS or USAXS, despite the different scattering geometries, wavelengths and scattering contrasts that apply. By combining SANS and USANS for different $\mathrm{H} / \mathrm{D}$ blends in the pore fluid it is possible, in principle, to discern the microstructure of the $\mathrm{CH}$ component within cement. This is because the formula and density for $\mathrm{CH}$ are well-known, the $\mathrm{OH}$ groups do not exchange to become OD, and a contrast match point occurs with the pore water at $32 \%$ $\mathrm{D}_{2} \mathrm{O}$. Thus, with these advances, the $\mathrm{CH}$ SANS component can be characterized over sufficient length scale to encompass both micrometer-scale $\mathrm{CH}$ crystals and fine nanoscale $\mathrm{CH}$. By subtracting out the $\mathrm{CH}$ SANS component, it is anticipated that the SANS associated with $\mathrm{C}-\mathrm{S}-\mathrm{H}$ will be investigated in future SAS studies over a contiguous scale range from $1 \mathrm{~nm}$ to $10 \mu \mathrm{m}$.

\section{Relationship of SANS to other neutron methods}

At wavelengths useful for the structural investigation of materials, neutrons have energies close to those for molecular thermal vibration (unlike X-rays which have much higher energies). Furthermore, the incoherent neutron scattering 
cross-section for hydrogen is particularly high. Thus, inelastic neutron scattering (INS) can measure molecular vibration spectra [26], particularly when hydrogen bonding or $\mathrm{OH}^{-}$ groups are involved as in $\mathrm{C}-\mathrm{S}-\mathrm{H}$ or $\mathrm{CH}$. Meanwhile, quasielastic neutron scattering (QENS) can exploit the effects of neutron recoil from a proton (of approximately the same mass as a neutron) in order to interrogate the free or bound state of water within $\mathrm{C}-\mathrm{S}-\mathrm{H}$ gel (or elsewhere within a cement) [23-25]. INS studies have recently provided significant information on the bonding within $\mathrm{C}-\mathrm{S}-\mathrm{H}$, as well as on the total $\mathrm{CH}$ formation, including nanoscale $\mathrm{CH}$ embedded in $\mathrm{C}-\mathrm{S}-\mathrm{H}[26]$. This contrasts with neutron and $\mathrm{X}$-ray diffraction methods that measure the micrometer scale crystalline $\mathrm{CH}$ formation. QENS measurements can also quantify the changes in the state of the cement pore water as it is incorporated into $\mathrm{C}-\mathrm{S}-\mathrm{H}$ gel and $\mathrm{CH}$ during the hydration process [25].

A comparison of the time dependences of the cement internal surface area deduced from the SANS Porod scattering (Fig. 4a), the bound water fraction (index) measured by QENS (Fig. 4b), and the heat output of the cement hydration reactions measured by thermal calorimetry (Fig. 4a), is illuminating. As with corresponding INS data (not shown), the QENS time dependence closely follows the hydration reaction heat output. This is to be expected given that both INS and QENS measure changes associated directly with hydration chemistry. However, while the microstructure changes measured by SANS or SAXS (increasing surface area and fractal microstructure development) follow the reaction chemistry through the time of the main hydration peak, this does not continue over more extended periods. The measurable microstructure changes in subtle ways after the first $24 \mathrm{~h}$, but the dense or inner-product $\mathrm{C}-\mathrm{S}-\mathrm{H}$ morphology produced from continued hydration is not visible to SANS or SAXS. This point distinguishes SAS methods from the other neutron and X-ray techniques, as well as methods like nuclear magnetic resonance (NMR) [28], which essentially measure the time dependence of the overall cement chemistry. By contrast, SANS or SAXS follow the development of the less dense or outer-product $\mathrm{C}-\mathrm{S}-\mathrm{H}$ morphology between the original clinker grains. It is this microstructural component that provides hydrated cement both with its strength and with its available (active) surface area for fluid sorption or foreign species adsorption throughout the cement service lifetime. This selectivity is an exploitable advantage of SAS methods in advanced cement research.

\section{Effects of $\mathrm{CaCl}_{2}$ and sucrose on the hydrated cement microstructure}

A useful application of SANS to cement paste is to investigate the microstructure differences that result from the use of various mineral and chemical additives. Here we discuss the effects of calcium chloride, a hydration accelerator, and sucrose (table sugar), a hydration retarder, on the hydrated microstructure of ordinary Portland cement (OPC) paste. The mechanisms by which these additives affect the kinetics are not fully understood (see discussion in [29]). Acceleration by $\mathrm{CaCl}_{2}$ is likely related to an increased rate of precipitation and floc-
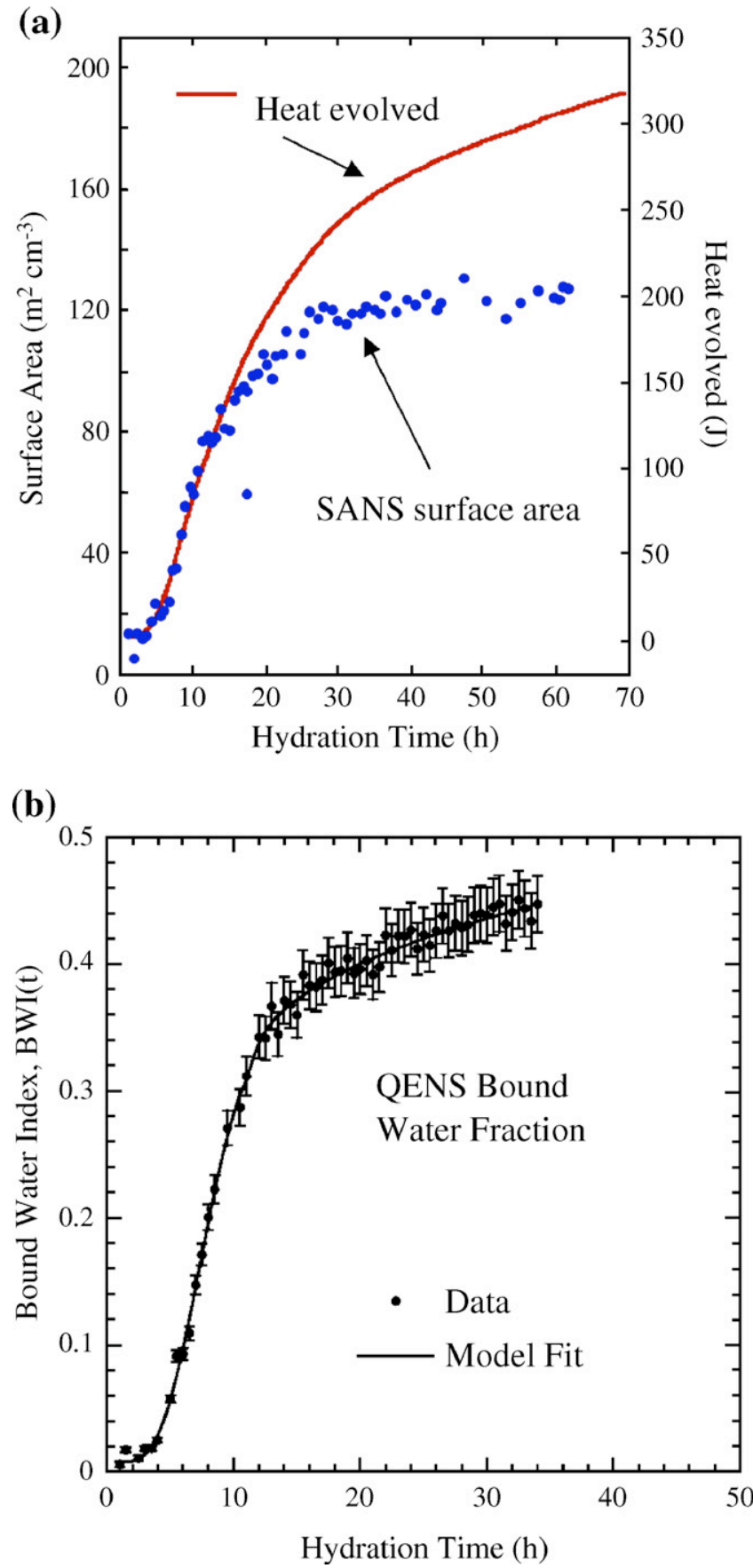

Fig. 4. Time dependence during cement hydration of: (a) SANS-measured surface area and reaction heat output [12], and (b) QENS-measured bound water fraction [23].

culation of $\mathrm{C}-\mathrm{S}-\mathrm{H}$ from solution, while sucrose is believed to adsorb onto calcium-containing hydration products, inhibiting further growth. Vollet and Craievich [17] previously used SAXS to study the effects of sugar and $\mathrm{CaCl}_{2}$ on the hydration kinetics of pure $\mathrm{C}_{3} \mathrm{~S}$, by using the change in the scattered intensity at a fixed $Q$ value to monitor the development of nanoporous hydration product. Whereas that study drew conclusions about the nanostructure of the early hydration product from an analysis of the kinetics, the present study uses the $I(Q)$ data from SANS to compare, directly, the microstructures of well-hydrated pastes. 
The $\mathrm{CaCl}_{2}$ was added in the amount of $2 \%$ mass OPC, and accelerated and control pastes with water-to-cement mass ratio $(\mathrm{w} / \mathrm{c})=0.4$ were hydrated for 28 days at $20^{\circ} \mathrm{C}$ under saturated conditions. As determined from thermal calorimetry, the $2 \%$ addition of $\mathrm{CaCl}_{2}$ reduced the time required to reach the maximum hydration rate from about $10 \mathrm{~h}$ to $3 \mathrm{~h}$, indicating that the early hydration kinetics are accelerated by a factor of about three. However, the addition of $\mathrm{CaCl}_{2}$ does not affect the degree of hydration of the paste at ages greater than a few days [29]. The sucrose was added in the amount of $1 \%$ mass OPC. Addition of sucrose significantly delays the start of hydration, so that the dormant induction period is increased from a few hours to several days or even weeks [30]. Once hydration starts, the hydration process proceeds at a normal or even slightly accelerated rate. To ensure that the induction period ended well before the age of 28 days, the sugar-retarded paste was hydrated at $40{ }^{\circ} \mathrm{C}$, as was an un-retarded control paste. Garci and Jennings [30] found that after 28 days at $40{ }^{\circ} \mathrm{C}$ the degree of hydration of the sugar-retarded paste was slightly higher than that of the control.

Fig. 5 shows the SANS data for the pastes described above, each cut into coupons $0.4 \mathrm{~mm}$ thick to avoid the multiple scattering effects sometimes observed for thicker samples [22]. Data were obtained using the NIST/NSF $30 \mathrm{~m}$ SANS at the NIST Center for Neutron Research. Addition of $\mathrm{CaCl}_{2}$ results in significant changes to the SANS data. The intensity at higher $Q$ values is increased, indicating more volume-fractal scattering, while the intensity at lower $Q$ values is decreased, such that the surface-fractal regime is apparent only at the lowest $Q$ values sampled. This is consistent with the formation of a greater amount of fine (nanoscale) $\mathrm{C}-\mathrm{S}-\mathrm{H}$ gel hydration product that fills the (micrometer-size) capillary pore space, leaving relatively little room for a distinguishable surface-fractal interfacial morphology on the capillary-pore walls. By comparison, the addition of sucrose generates relatively minor changes to the SANS data, the only apparent difference being a modest decrease in the surface-fractal component in the scattering.
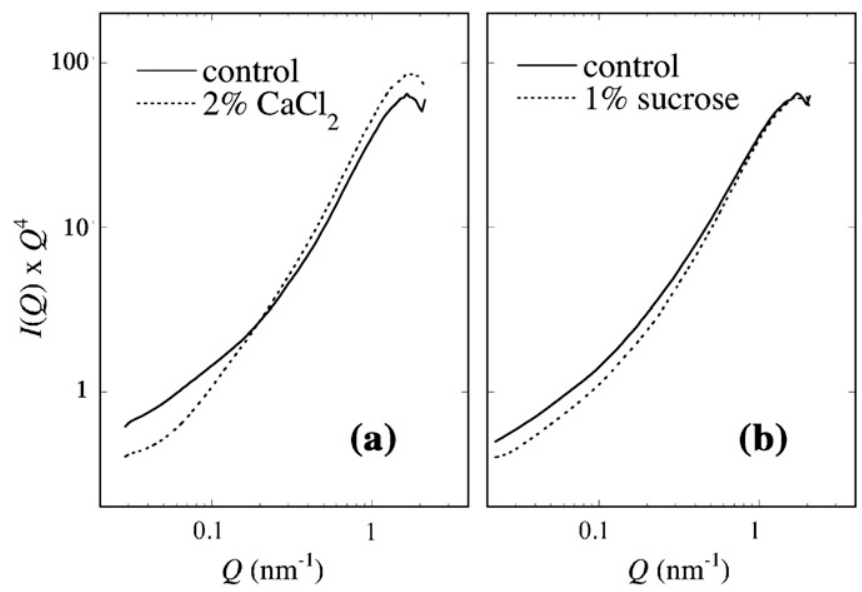

Fig. 5. SANS data for OPC pastes hydrated with and without additives, plotted as $I(Q) \times Q^{4}$ versus $Q$. All pastes were mixed at $\mathrm{w} / \mathrm{c}=0.4$ and cured for 28 days. (a) Addition of $2 \% \mathrm{CaCl}_{2}$ (pastes cured at $20{ }^{\circ} \mathrm{C}$ ). (b) Addition of $1 \%$ sucrose (pastes cured at $40^{\circ} \mathrm{C}$ ).
Table 1

Microstructure parameters derived from fractal model fits to the SANS data shown in Fig. 5

\begin{tabular}{lcccc}
\hline & $\begin{array}{l}\text { Control } \\
\left(20{ }^{\circ} \mathrm{C}\right)\end{array}$ & $\begin{array}{l}2 \% \mathrm{CaCl}_{2} \\
\left(20^{\circ} \mathrm{C}\right)\end{array}$ & $\begin{array}{l}\text { Control } \\
\left(40{ }^{\circ} \mathrm{C}\right)\end{array}$ & $\begin{array}{l}1 \% \text { sucrose } \\
\left(40{ }^{\circ} \mathrm{C}\right)\end{array}$ \\
\hline$S_{\mathrm{T}}\left(\mathrm{m}^{2} / \mathrm{cm}^{3}\right)$ & $96(5)$ & $133(7)$ & $101(5)$ & $98(5)$ \\
$a_{\mathrm{S}}(\mathrm{nm})$ & $4.15(12)$ & $4.05(12)$ & $4.06(12)$ & $4.15(12)$ \\
$\phi_{\mathrm{CSH}}$ & $0.109(6)$ & $0.150(8)$ & $0.129(6)$ & $0.099(5)$ \\
$\eta$ & $0.575(17)$ & $0.586(17)$ & $0.614(18)$ & $0.564(17)$ \\
$D_{\mathrm{V}}$ & $2.61(5)$ & $2.60(5)$ & $2.58(5)$ & $2.54(5)$ \\
\hline
\end{tabular}

The numbers in parentheses are estimated standard deviations in least significant digits.

Table 1 lists some microstructure parameters obtained by fitting models to the data shown in Fig. 5. The total surface area, $S_{\mathrm{T}}$, was obtained from the Porod scattering at the highest $Q$ values using Eq. (2). The other parameters were obtained by applying the fractal model discussed earlier $[4,5,10]$. We follow the fractal model interpretation and contrast assumptions of our recent previous work [15], which does not take account of the ongoing work to distinguish between the fine $\mathrm{CH}$ and $\mathrm{C}-\mathrm{S}-\mathrm{H}$ morphologies mentioned above. However, it suffices for the microstructure comparisons made here between accelerated and retarded cement systems. In Table 1, the parameter $a_{\mathrm{S}}$ is the fitted sphere-equivalent particle diameter of the basic volumefractal building blocks, and $\eta$ is the fitted local packing fraction of these particles. Parameter $\phi_{\mathrm{CSH}}$ is the fitted volume fraction of solid $\mathrm{C}-\mathrm{S}-\mathrm{H}$ gel in the paste accessible to SANS, which, as discussed earlier, includes only the low-density "outer product" form of $\mathrm{C}-\mathrm{S}-\mathrm{H}$. Finally, $D_{\mathrm{V}}$ is the fitted volume-fractal exponent.

From Table 1 it can be seen that the surface areas of the control and sucrose-retarded pastes are similar, while that of the $\mathrm{CaCl}_{2}$ accelerated paste is some $30 \%$ higher. A similar trend is observed in the $\mathrm{C}-\mathrm{S}-\mathrm{H}$ volume fraction, so it can be concluded that $\mathrm{CaCl}_{2}$ increases the amount of observable lowdensity $\mathrm{C}-\mathrm{S}-\mathrm{H}$. This is in agreement with the results of Vollet and Craievich [17], who found that the degree of hydration at the end of the early rapid hydration period (associated with formation of fine hydration product) is significantly increased by $\mathrm{CaCl}_{2}$, and slightly reduced by sugar. It can be inferred that, since the degree of hydration is not increased, $\mathrm{CaCl}_{2}$ decreases the amount of high-density $\mathrm{C}-\mathrm{S}-\mathrm{H}$. The results are in good agreement with the observation that $\mathrm{CaCl}_{2}$ accelerated pastes undergo more drying shrinkage, as the low density $\mathrm{C}-\mathrm{S}-\mathrm{H}$ gel is the primary shrinking phase. In all pastes the particle diameter, $a_{\mathrm{S}}$, is essentially constant, and the variations in $\eta$ and $D_{\mathrm{V}}$ are relatively small, indicating that the nanoscale $\mathrm{C}-\mathrm{S}-\mathrm{H}$ gel morphology is not significantly affected by the additives.

\section{Conclusions and issues for further consideration}

In this paper we have indicated how recent developments in SAXS, SANS, INS, and QENS methods for studying hydrating cement systems can be used to elucidate the morphology of the $\mathrm{C}-\mathrm{S}-\mathrm{H}$ gel over an extended length scale. A number of questions can now be made the subject of further investigation: How 
is the $\mathrm{C}-\mathrm{S}-\mathrm{H}$ globule/fractal picture of $\mathrm{C}-\mathrm{S}-\mathrm{H}$ associated with SAXS and SANS to be reconciled with the various other particulate and sheet morphologies associated with characterization methods such as electron microscopy [19]? What is the dense or inner product morphology of $\mathrm{C}-\mathrm{S}-\mathrm{H}$ and why is it virtually invisible to SAXS or SANS? To what extent and under what conditions can inner product and outer product $\mathrm{C}-\mathrm{S}-\mathrm{H}$ transform between each other? Our knowledge and understanding of cements and $\mathrm{C}-\mathrm{S}-\mathrm{H}$ gel are now sufficient that it is timely to consider how such questions might be answered in future studies.

We have also illustrated the application of SANS to quantify the effects of an accelerator $\left(\mathrm{CaCl}_{2}\right)$ and a retarder (sucrose) on the hydrated microstructure. The use of $\mathrm{CaCl}_{2}$ did not affect the nanometer-scale volume fractal structure of the $\mathrm{C}-\mathrm{S}-\mathrm{H}$ gel, as determined from the building-block particle size and fractal exponent, but did significantly increase both the total internal surface area and the volume fraction of the specimen occupied by low-density $\mathrm{C}-\mathrm{S}-\mathrm{H}$ visible to SANS. The latter observations are in agreement with the known tendency for $\mathrm{CaCl}_{2}$ to increase drying shrinkage. For the curing conditions used here, sucrose had no measurable effect on the mature microstructure despite its strong retarding properties.

\section{Acknowledgements}

The work performed at Northwestern University was supported by the National Science Foundation under contract CMS-0409571. The work at NIST utilized facilities supported in part by the National Science Foundation under Agreement No. DMR-9986442.

\section{References}

[1] D.N. Winslow, S. Diamond, Specific surface of hardened cement paste as determined by small-angle X-ray scattering, J. Am. Ceram. Soc. 57 (1974) 193-197.

[2] A.J. Allen, C.G. Windsor, V.S. Rainey, D. Pearson, D.D. Double, N.McN. Alford, A small-angle scattering study of cement porosities, J. Phys. D 15 (1982) $1817-1833$.

[3] J.J. Volkl, R.E. Beddoe, M.J. Setzer, The specific surface of hardened cement paste by small-angle X-ray-scattering-effect of moisture-content and chlorides, Cem. Concr. Res. 17 (1987) 81-88.

[4] A.J. Allen, R.C. Oberthur, D. Pearson, P. Schofield, C.R. Wilding, Development of the fine porosity and gel structure of hydrating cement systems, Philos. Mag., B 56 (1987) 263-288.

[5] A.J. Allen, Time-resolved phenomena in cements, clays and porous rocks, J. Appl. Crystallogr. 24 (1991) 624-634.

[6] F. Eichhorn, F. Haussler, H. Baumbach, Structural studies on hydrating cement pastes, J. Phys. IV 3 (C8) (1993) 369-372.

[7] D.N. Winslow, J.M. Bukowski, J.F. Young, The early evolution of the surface of hydrating cement, Cem. Concr. Res. 24 (1994) 1025-1032.

[8] R.E. Beddoe, K. Lang, Effect of moisture on fractal dimension and specific surface of hardened cement paste by small-angle X-ray-scattering, Cem. Concr. Res. 24 (1994) 605-612.

[9] D. Winslow, J.M. Bukowski, J.F. Young, The fractal arrangement of hydrated cement paste, Cem. Concr. Res. 25 (1995) 147-156.
[10] A.J. Allen, R.A. Livingston, Relationship between differences in silica fume additives and fine-scale microstructural evolution in cement based materials, Adv. Cem. Based Mater. 8 (1998) 118-131.

[11] J.J. Thomas, H.M. Jennings, A.J. Allen, Determination of the neutron scattering contrast of hydrated Portland cement paste using $\mathrm{H}_{2} \mathrm{O} / \mathrm{D}_{2} \mathrm{O}$ exchange, Advn. Cem. Based Mater. 7 (1998) 119-122.

[12] J.J. Thomas, H.M. Jennings, A.J. Allen, The surface area of cement paste as measured by neutron scattering - evidence for two $\mathrm{C}-\mathrm{S}-\mathrm{H}$ morphologies, Cem. Concr. Res. 28 (1998) 897-905.

[13] J.J. Thomas, H.M. Jennings, A.J. Allen, The surface area of hardened cement paste as measured by various techniques, Concr. Sci. Eng. 1 (1999) 45-64.

[14] F. Gaboriaud, A. Nonat, D. Chaumont, A. Craievich, B. Hanquet, Si-29 NMR and small-angle X-ray scattering studies of the effect of alkaline ions $\left(\mathrm{Li}^{+}, \mathrm{Na}+\right.$, and $\left.\mathrm{K}+\right)$ in silico-alkaline sols, J. Phys. Chem., B 103 (1999) 2091-2099.

[15] J.J. Thomas, J.J. Chen, A.J. Allen, H.M. Jennings, Effects of decalcification on the microstructure and surface area of cement and tricalcium silicate pastes, Cem. Concr. Res. 34 (2004) 2297-2307.

[16] M. Kriechbaum, G. Degovics, J. Tritthart, P. Laggner, Fractal structure of Portland cement paste during age hardening analyzed by small-angle X-ray scattering, Prog. Colloid \& Polym. Sci. 79 (1989) 101-105.

[17] D.R. Vollet, A.F. Craievich, Effects of temperature and of the addition of accelerating and retarding agents on the kinetics of hydration of tricalcium silicate, J. Phys. Chem., B 104 (2000) 12143-12148.

[18] G. Porod, General theory, in: O. Glatter, O. Kratky (Eds.), Small-Angle X-ray Scattering, Academic Press, London, 1982, pp. 17-51.

[19] I.G. Richardson, Tobermorite/jennite- and tobermorite/calcium hydroxidebased models for the structure of $\mathrm{C}-\mathrm{S}-\mathrm{H}$ : applicability to hardened pastes of tricalcium silicate, beta-dicalcium silicate, Portland cement, and blends of Portland cement with blast-furnace slag, metakaolin, or silica fume, Cem. Concr. Res. 34 (2004) 1733-1777.

[20] A.R. Drews, J.G. Barker, C.J. Glinka, M. Agamalian, Development of a thermal-neutron double-crystal diffractometer for USANS at NIST, Physica. B 241 (1997) 189-191.

[21] J.G. Barker, C.J. Glinka, J.J. Moyer, M.H. Kim, A.R. Drews, M. Agamalian, Design and performance of a thermal-neutron double-crystal diffractometer for USANS at NIST, J. Appl. Crystallogr. 38 (2005) 1004-1011.

[22] J.W. Phair, J.C. Schulz, W. Bertram, L.P. Aldridge, Investigation of the microstructure of alkali-activated cements by neutron scattering, Cem. Concr Res. 33 (2003) 1811-1824.

[23] J.J. Thomas, D.A. Neumann, S.A. FitzGerald, R.A. Livingston, State of water in hydrating tricalcium silicate and Portland cement pastes as measured by quasi-elastic neutron scattering, J. Am. Ceram. Soc. 84 (2001) 1811-1816.

[24] A.J. Allen, J.C. McLaughlin, D.A. Neumann, R.A. Livingston, In situ quasi-elastic scattering characterization of particle size effects on the hydration of tricalcium silicate, J. Mater. Res. 19 (2004) 3242-3254.

[25] E. Fratini, S.-H. Chen, P. Baglioni, M.-C. Bellissent-Funel, Dynamic scaling of quasielastic neutron scattering spectra from interfacial water, Phys. Rev., E Stat. Phys. Plasmas Fluids Relat. Interdiscip. Topics 64 (2001) 020201

[26] J.J. Thomas, J.J. Chen, H.M. Jennings, D.A. Neumann, Ca-OH bonding in the $\mathrm{C}-\mathrm{S}-\mathrm{H}$ gel phase of tricalcium silicate and white portland cement pastes measured by inelastic neutron scattering, Chem. Mater. 15 (2003) 3813-3817.

[27] A.J. Allen, Characterization of ceramics by X-ray and neutron small-angle scattering, J. Am. Ceram. Soc. 88 (2005) 1367-1381.

[28] C. Porteneuve, J.P. Korb, D. Petit, H. Zanni, Structure-texture correlation in ultra-high-performance concrete - a nuclear magnetic resonance study, Cem. Concr. Res. 32 (2002) 97-101.

[29] H.F.W. Taylor, Cement Chemistry, 2nd Ed.Thomas Telford, London, 1997.

[30] M.C. Garci Jueger, H.M. Jennings, New insights into the effect of sugar on the hydration and microstructure of cement pastes, Cem. Concr. Res. 32 (2002) 393-399. 